\title{
Correction to: Numerical Simulation of the Time-Dependent Mild-Slope Equation by the Generalized Finite Difference Method
}

\author{
Ting Zhang, ${ }^{1}$ Zhen-Huan Lin, ${ }^{1}$ Chuan Lin, ${ }^{1}$ (D) Lin Liang, ${ }^{1}$ and Chia-Ming Fan ${ }^{2}$
}

Correction to: Pure Appl. Geophys. 178 (2021), 4401-4424

https://doi.org/10.1007/s00024-021-02870-4

The original version of this paper was inadvertently published with an incorrect affiliation for the author Chia-Ming Fan.

The correct affiliation is:

Department of Harbor and River Engineering and Computation and Simulation Center, National Taiwan Ocean University, Keelung 20224, Taiwan, China.

The original article has been corrected. We apologise for any inconvenience caused to our readers.

1 Department of Water Resources and Harbor Engineering, College of Civil Engineering, Fuzhou University, Fuzhou, China. E-mail: linchuan@fzu.edu.cn

2 Department of Harbor and River Engineering and Computation and Simulation Center, National Taiwan Ocean University, Keelung 20224, Taiwan, China. 